\title{
Impact of a Remote Monitoring Programme Including Lifestyle Education Software in Type 2 Diabetes: Results of the Educ@dom Randomised Multicentre Study
}

\author{
Marie-Christine Turnin (i) $\cdot$ Pierre Gourdy $\cdot$ Jacques Martini $\cdot$ Jean-Christophe Buisson • \\ Marie-Christine Chauchard · Jacqueline Delaunay · Solène Schirr-Bonnans · Soumia Taoui · \\ Marie-France Poncet • Valeria Cosma $\cdot$ Sandrine Lablanche $\cdot$ Magali Coustols-Valat $\cdot$ Lucie Chaillous · \\ Charles Thivolet $\cdot$ Caroline Sanz $\cdot$ Alfred Penfornis $\cdot$ Benoît Lepage $\cdot$ Hélène Colineaux $\cdot$ \\ Michaël Mounié $\cdot$ Nadège Costa $\cdot$ Laurent Molinier · Hélène Hanaire on behalf of the Educ@dom Study Group
}

Received: March 31, 2021 / Accepted: June 5, 2021 / Published online: June 23, 2021

(c) The Author(s) 2021

\section{ABSTRACT}

Introduction: Telemonitoring in type 2 diabetes (T2D) is mainly based on glucose monitoring. A new type of connected device which routinely gathers data on weight, physical

M.-C. Turnin $(\bowtie) \cdot$ P. Gourdy · J. Martini ·

M.-C. Chauchard · J. Delaunay ·

S. Schirr-Bonnans $\cdot$ S. Taoui $\cdot$ H. Hanaire

Department of Diabetology, Metabolic Diseases and

Nutrition, University Hospital of Toulouse, 1

Avenue Jean Poulhès, TSA 50032, Toulouse cedex 9, 31059 Toulouse, France

e-mail: turnin.mc@chu-toulouse.fr

P. Gourdy · H. Hanaire

Institute of Metabolic and Cardiovascular Diseases, UMR1297 INSERM/UPS, Toulouse University,

Toulouse, France

J. Martini · M.-C. Chauchard

DIAMIP Network, Toulouse, France

J.-C. Buisson

ENSEEIHT, Toulouse, France

M.-F. Poncet

Claude Bernard Clinic, Albi, France

V. Cosma

Nîmes Hospital Centre, Nimes, France

S. Lablanche

University Hospital, Grenoble, France activity and food intake could improve patients' diabetes control. The main aim of this study was to assess the efficacy of an at-home interventional programme incorporating such devices and lifestyle education software on diabetes control, i.e., change in HbA1c, compared to standard care.

\section{Coustols-Valat}

Ambroise Paré Clinic, Toulouse, France

L. Chaillous

University Hospital, Nantes, France

C. Thivolet

University Hospital, Lyon, France

C. Sanz

Pasteur Clinic, Toulouse, France

\section{A. Penfornis}

Sud Francilien Hospital, Corbeil-Essonnes and ParisSaclay University, Paris, France

\section{B. Lepage $\cdot H$. Colineaux}

University Department of Epidemiology, Health Economics and Public Health, University Hospital, Toulouse, France

M. Mounié · N. Costa $\cdot$ L. Molinier

Medico-Economic Unit, Medical Information Department, University Hospital, Toulouse, France 
Methods: This multicentre study randomly assigned 282 people with T2D to either a telemonitoring group (TMG) or a control group (CG) for a 1-year intervention period. While routine follow-up was maintained in the CG, TMG subjects were provided with interactive lifestyle educational software (with artificial intelligence algorithms) and connected objects (blood glucose meters, scales and actimeters) for use in their own homes and were remotely monitored by their diabetologists. Changes in $\mathrm{HbA}_{1 \mathrm{c}}$ were compared between groups using a mixed linear model.

Results: The mean $\mathrm{HbA}_{1 \mathrm{c}}$ dropped from $7.8 \pm 0.8 \% \quad(62 \mathrm{mmol} / \mathrm{mol}) \quad$ to $7.4 \pm 1.0 \%$ $(57 \mathrm{mmol} / \mathrm{mol})$ in the TMG and from $7.8 \pm 0.8 \% \quad(62 \mathrm{mmol} / \mathrm{mol}) \quad$ to $7.6 \pm 1.0 \%$ $(60 \mathrm{mmol} / \mathrm{mol})$ in the $\mathrm{CG}$, resulting in an intergroup difference of $-0.16(p=0.06)$ in favour of TMG, after adjustment for confounding factors. Within TMG, the decrease in $\mathrm{HbA}_{1 \mathrm{c}}$ was greater in frequent users: $-0.23 \%$ $(p=0.03)$ in the case of connections to telemonitoring synthesis above the median and $0.21 \%(p=0.05)$ in the case of connections to tele-education software above the median compared to the CG. Significant weight loss was observed in the TMG but only in women $(p=0.01)$.

Findings: The EDUC@DOM telemonitoring and tele-education device did not highlight a significant decrease in HbA1c levels compared to routine management although a slight, albeit significant improvement in glycaemic control was observed in the frequent user subgroup as well as significant weight loss but only in women. A high level of satisfaction with the connected device was recorded amongst all participants.

Trial Registration: This trial was registered in the Clinical Trials Database on September 27, 2013, under no. NCT01955031 and bears IDRCB number 2013-A00391-44.

Keywords: Type 2 diabetes; Lifestyle management; Glucose control; Telemonitoring; Tele-education; Health-related objects

\section{Key Summary Points}

This work is one of the first to use a telemonitoring device which routinely gathers data on weight, physical activity and diet in addition to glycaemia control in order to improve the monitoring of type 2 diabetes patients

The device used is connected to interactive lifestyle educational software based on artificial intelligence algorithms

The telemonitoring and tele-education device failed to show a significant fall in HbA1c levels in individuals with type 2 diabetes $(p=0.06)$ despite a slight, albeit significant improvement in the frequent user subgroup. A high level of satisfaction was recorded among all participants

Significant weight loss was observed in the telemonitoring group but only in women

\section{DIGITAL FEATURES}

This article is published with digital features, including a summary slide, to facilitate understanding of the article. To view digital features for this article go to https://doi.org/10.6084/ m9.figshare.14732148.

\section{INTRODUCTION}

According to current guidelines, first-line intervention in individuals living with type 2 diabetes (T2D) is based on lifestyle changes aimed at a balanced diet and frequent physical activity in order to achieve better glycaemic control and, if necessary, weight reduction [1]. Such lifestyle modifications need to be sustained with a long-term perspective, regardless of the combined pharmacological treatment regimens. Although many studies have demonstrated the ability of lifestyle interventions to improve glycaemic control in $\mathrm{T} 2 \mathrm{D}$, this 
beneficial metabolic effect is difficult to sustain long term [2-4]. Technological innovations, including the use of health-related objects, currently make it possible to remotely monitor individuals with diabetes in their own homes. Close intervention in their daily lives is thus feasible, both as a support towards improving lifestyle and adapting treatment [5-20].

This randomised study was based on the hypothesis that tele-education and telemonitoring improve lifestyle and quality of glycaemic control in patients with T2D while rationalising the need for face-to-face consultations. The long-term goal is to reduce the incidence of diabetic complications and costs. We designed and developed Nutri-Educ, a new athome education software, in order to support changes in lifestyle. Nutri-Educ is the only software including artificial intelligence algorithms, which allow it to deliver personalised advice to correct errors in meal composition. This software has been combined with the use of connected objects to offer an integrated telemonitoring programme [21]. Changes in $\mathrm{HbA}_{1 \mathrm{c}}$, weight and waist circumference have been reviewed over a 1-year period in T2D subjects equipped with this innovative telemonitoring programme compared to standard care. The main aim of this study was to assess the efficacy of the telemonitoring programme in improving glycaemic control, i.e., decreasing $\mathrm{HbA}_{1 \mathrm{c}}$ levels compared to standard care.

\section{METHOD}

\section{Trial Design}

This open-label, randomised, controlled trial with two parallel arms, telemonitoring group (TMG) and control group (CG), involved 16 investigation sites: 5 university teaching hospitals, 1 non-teaching hospital centre, a diabetes care network linking 8 public and private health establishments and 2 private practice physicians.

\section{Participants}

The subjects enrolled in the study were aged 18 years or above, had a documented medical history of $\mathrm{T} 2 \mathrm{D}$, with or without insulin treatment, and with a recent $\mathrm{HbA}_{1 \mathrm{c}}$ value $>6.5 \%$ $(48 \mathrm{mmol} / \mathrm{mol}) \quad$ and $\leq 10 \% \quad(86 \mathrm{mmol} / \mathrm{mol})$. They had to have an active internet connection at home. People with severe or active comorbidities, reduced mobility, serious eating disorders or who had undergone bariatric surgery were excluded [21].

\section{Interventions}

The home telemonitoring device combined biomedical data sensors (scale with impedancemetry, actimeter and blood glucose meter) with educational software [22, 23] available on tablets. Three tele-educational software programmes were given to participants in the TMG: Nutri-Kiosk-a set of self-learning quizzes for formative assessment based on nutritional knowledge; Acti-Kiosk-support in starting physical activity (assessment, information and videos); Nutri-Educ - a personalised nutritional educational software application that meets international clinical recommendations [24]. It was developed using artificial intelligence algorithms and has already demonstrated its beneficial impact in obesity and T2D patients [25-27]. Nutri-Educ was designed to help patients in the TMG group to improve their nutritional balance. The patients had to enter meal composition details into the software, which subsequently analysed the meals and proposed corrections taking into account the patients' individual profiles, dietary habits and tastes. A more detailed description of the device has already been published [21].

The monitoring interface linking TMG participants with investigators was designed as a secure web platform receiving all data from connected objects. TMG subjects sent these data to the platform on a weekly basis and had free access to this information and to their monitoring summary. The frequency of data consultation by investigators was left to their discretion. However, according to the protocol, 
they were sent an email every month to encourage them to carry out the telemonitoring. A summary of all data recorded over the last 30 days was available for rapid interpretation of results in order to facilitate follow-up and the telemonitoring process. A break-down of the monthly changes also provided an overview of the progress in the monitored parameters. Modifiable alerts notified investigating physicians about certain events including hypoglycaemia, hyperglycaemic tendencies and significant weight gain or loss. Investigators had access to patient records to personalise the follow-up by modulating blood glucose, body weight and physical activity targets to be achieved over time as well as alert levels according to each participant's profile. A secured messaging system allowed repeated and interactive discussions with telemonitored individuals to advise, encourage and coach them. Investigating physicians were required to provide a report on telemonitoring and the actions implemented in order to validate the telemonitoring process and ensure traceability of the action taken. General practitioners were given regular telemonitoring progress reports.

Telemonitoring follow-up for the TMG was planned over a 12-month period, with no faceto-face consultation scheduled in advance. Follow-up in the CG included face-to-face consultations in accordance with standard practices.

\section{Outcomes}

The primary endpoint was the intergroup change in glycated haemoglobin levels $\left(\mathrm{HbA}_{1 \mathrm{c}}\right)$ over the 12-month intervention period. Changes in other clinical parameters (body weight, BMI and waist circumference) were also considered and compared between groups in the overall population and then taking gender into account. Compliance with the device, including frequency of use and its impact, was assessed. Finally, the satisfaction of individuals with diabetes and caregivers was also recorded using an ad hoc questionnaire dedicated to the device in question.

\section{Randomisation}

The individuals with T2D enrolled in the trial were randomly assigned to two groups (telemonitoring group [TMG] or control group [CG]) balanced by random block sizes and stratified by $\mathrm{HbA}_{1 \mathrm{c}}$ level at inclusion [threshold: 7.5\% $(58 \mathrm{mmol} / \mathrm{mol})]$, which was a priori expected to be the most relevant factor associated with changes in HbA1c levels during intervention.

\section{Sample Size}

The sample size was initially determined to detect a $0.5 \mathrm{mmol} / \mathrm{mol}$ difference in the primary efficacy endpoint over 12 months, with a power of $80 \%$ and a $5 \%$ type I error (2-tailed test). We applied the Frison and Pocock approach [28], based on a comparison of the mean of four repeated measurements between the two intervention arms. A sample size of 234 (117 per arm) was necessary, plus $20 \%$ to avoid loss of power due to loss of follow-up, resulting in a total of 282 subjects to be recruited (141 per arm).

\section{Statistical Analysis}

An intention-to-treat effectiveness analysis was carried out. Since the analysis was performed on complete data (no imputation for missing data), the intention-to-treat population comprised all randomised participants having at least one $\mathrm{HbA}_{1 \mathrm{c}}$ value collected during follow-up (primary endpoint) and having no missing data regarding the confounding factors defined a priori. The proportion of subjects randomised but not analysed was $<10 \%$.

Concerning the primary efficacy endpoint, we compared the two groups using a linear mixed-effects model for repeated measures 
(MMRM), modelling all $\mathrm{HbA}_{1 \mathrm{c}}$ measurements performed during the 1-year intervention period. To ensure the comparability of the two groups (TMG versus CG) and to enhance statistical power, the model was adjusted for the confounding factors defined a priori (baseline $\mathrm{HbA}_{1 \mathrm{c}}$, age, gender, diabetes complications, obesity, duration of diabetes and insulin treatment). The search for an interaction between the time of measurement and the intervention group was carried out as well as a centre effect. Additional analyses taking patient compliance (number of connections) into account were also performed. For this purpose, we considered the number of connections to data synthesis and to Nutri-Educ software, respectively. These variables were categorised into two groups based on the median value. We therefore classified the most assiduous users into TMGs + group (number of connections to the synthesis above the median) and TMGn+ group (number of connections to the Nutri-Educ software above the median).

Changes in body weight, BMI and waist circumference during follow-up were compared using the same procedure. The analyses were also stratified by gender to assess a potential change in terms of effect.

Since the primary and secondary analyses suggested an effect dependent on compliance with the device, the correlation between the changes in $\mathrm{HbA}_{1 \mathrm{c}}$ and $\mathrm{BMI}$ was tested according to the frequency of connections to the device (synthesis and Nutri-Educ) in the TMG.

Data analysis was performed using Stata software (Statistical Software: Release 14.0. Stata Corporation, College Station, TX).

\section{Ethics Approval and Consent to Participate}

All T2D patients participating in this research received verbal and written information about the study and were given the opportunity to ask any questions to help them understand the study. They signed a voluntary informed consent form before the research began. This study was submitted to the South-West and French Overseas Territories' Research Ethics Committee
(REC) [Comité de Protection des Personnes (CPP)] and received a favourable opinion from the REC on $27 / 05 / 2013$.

\section{RESULTS}

\section{Patient Disposition and Baseline Characteristics}

Two hundred eighty-two T2D patients were enrolled in the study according to baseline $\mathrm{HbA}_{1 \mathrm{c}}$ level stratification, i.e., $\mathrm{HbA}_{1 \mathrm{c}}<7.5 \%$ $(58 \mathrm{mmol} / \mathrm{mol}) \quad(n=131) \quad$ or $\quad \mathrm{HbA}_{1 \mathrm{c}} \geq 7.5 \%$ $(n=151)$. They were then randomly assigned to two groups of 141 participants for either telemonitoring (TMG) or standard (CG) management. Nineteen participants were lost to followup (13 in the TMG and six in the CG) (Fig. 1).

The analysis set comprised 263 individuals (128 in the TMG and 135 in the CG) who had at least one $\mathrm{HbA}_{1 \mathrm{c}}$ measurement during the follow-up period. Multivariate analyses were based on 256 participants (126 in the TMG and 130 in the CG) with no missing data among the variables entered in the model (data and confounding factors defined a priori). Finally, 242 individuals presented with an $\mathrm{HbA}_{1 \mathrm{c}}$ measurement at 12 months, i.e., at the end of the intervention period.

The baseline characteristics of the study population show no difference between the two groups (Table 1).

\section{Frequency of Device Use in Telemonitored Patients}

In the TMG, the mean number of connections to the device by patient was $104 \pm 78$ (median value: 86 ) over the 12 -month follow-up period, which corresponds to a frequency of about twice a week. Users could access the different device functions at each connection. Mean data synthesis (TMGs) and Nutri-Educ software (TMGn) access figures were $44 \pm 49$ (median value: 29 ) and $48 \pm 61$ (median value: 31 ), respectively, demonstrating almost weekly use. On average, TMG patients sent $14 \pm 13$ messages (median value: 11) to the investigators, 
i.e., about one message per month. The mean messaging frequency from the investigators to the participants was $5 \pm 5$ (median value: 3 ).

\section{Primary Outcome: Changes in HbA1c over the Intervention Period}

During the 12-month intervention period, the mean HbA1c level dropped from $7.8 \pm 0.8 \%$ $(62 \mathrm{mmol} / \mathrm{mol})$ to $7.4 \pm 1.0 \%(57 \mathrm{mmol} / \mathrm{mol})$ in the TMG and from $7.8 \pm 0.8 \%$ $(62 \mathrm{mmol} / \mathrm{mol})$ to $7.6 \pm 1.0 \%(60 \mathrm{mmol} / \mathrm{mol})$ in the CG. Among individuals with measurements available at 12 months, no significant decrease in the mean HbA1c level was recorded in the TMG compared to the CG: - 0.19\% (95\% CI $[-0.43 ; 0.05], p=0.12)$. Moreover, the remaining difference between HbA1c value achieved at 12 months and the target initially set with the investigator (available for 241 participants) was not significantly lower in the TMG compared to the CG $(-0.13 \%,[-0.37$; $0.10], p=0.27$ ).

After adjustment for confounding factors, the intergroup difference was $-0.16 \%$ (95\% CI $[-0.32 ; 0.01], p=0.06)$, in favour of TMG (Fig. 2). The change predicted by the adjusted model showed an improvement in HbA1c level from T0 to T12: 7.75\% (95\% CI [7.65; 7.85]) $(61 \mathrm{mmol} / \mathrm{mol})$ to $7.49 \%(95 \%$ CI $[7.31 ; 7.66])$ (58 $\mathrm{mmol} / \mathrm{mol})$ in the TMG and to $7.64 \%$ (95\% CI $[7.47 ; 7.82])(60 \mathrm{mmol} / \mathrm{mol})$ in the CG.

Taking into account stratification on baseline $\mathrm{HbA1c}$, the intergroup difference in terms of change was $-0.13(95 \%$ CI $[-0.32 ; 0.05]$, $p=0.16)$ in the lower stratum (HbA1c $<7.5 \%$, $n=120)$, and -0.19 (95\% CI [-0.45; 0.07], $p=0.16)$ in the upper stratum (HbA1c $\geq 7.5 \%$, $n=136)$. We found no interaction between baseline HbA1c stratum and telemonitoring intervention regarding changes in HbA1c levels $(p=0.55)$.

Individuals characterised as TMGs+ (connections to telemonitoring synthesis above the median) exhibited a greater decrease in HbA1c compared to the CG: $-0.23 \% \quad(95 \%$ CI $[-0.43 ;-0.03], p=0.03)$, with a 12-month predicted change of $-0.31 \% \quad(95 \% \quad \mathrm{CI}$ $[-0.53 ;-0.10])$ in TMGs+ versus $-0.08 \%$
[-0.26; 0.09] in CG, moving from $7.75 \%$ $(61 \mathrm{mmol} / \mathrm{mol})$ to $7.42 \%(58 \mathrm{mmol} / \mathrm{mol})$ for TMGs+ versus $7.64 \%(60 \mathrm{mmol} / \mathrm{mol})$ for CG. Similarly, a fall in HbA1c levels was more important in TMGn+ (connections to NutriEduc above the median) than in CG participants: $-0.21 \% \quad(95 \% \quad$ CI $\quad[-0.41 ;-0.00])$, $p=0.05)$, with a 12 -month predicted decrease of $-0.29 \% \quad(95 \% \quad$ CI $\quad[-0.51 ;-0.07])$ in TMGn+ versus $-0.09 \%[-0.26 ; 0.09]$ in CG, moving from $7.75 \%(61 \mathrm{mmol} / \mathrm{mol})$ to $7.44 \%$ $(58 \mathrm{mmol} / \mathrm{mol})$ for $\mathrm{TMGn}+$ versus $7.64 \%$ (60 mmol $/ \mathrm{mol}$ ) for GC (Table 2).

\section{Secondary Outcomes: Changes in BMI and Waist Circumference During the Intervention Period}

Among individuals with data available over 12 months $(n=240)$, BMI decreased by $0.30 \pm 1.9 \mathrm{~kg} / \mathrm{m}^{2}+$ in the TMG and increased by $0.06 \pm 1.3 \mathrm{~kg} / \mathrm{m}^{2}$ in the CG resulting in a non-significant intergroup difference of $-0.36 \mathrm{~kg} / \mathrm{m}^{2} \quad(95 \% \quad$ CI $\quad[-0.76 ; 0.05]$, $p=0.08)$ in favour of the TMG. After adjustment for confounding factors $(n=236)$, the average predicted difference between the two groups was $-0.30 \mathrm{~kg} / \mathrm{m}^{2}, \quad(95 \%$ CI $[-0.70$; 0.09], $p=0.14$ ) with an expected 12-month change of $-0.24 \mathrm{~kg} / \mathrm{m}^{2}$ in the TMG versus $0.06 \mathrm{~kg} / \mathrm{m}^{2}$ in the CG. The expected BMI changes between baseline and the end of intervention were $-0.40 \mathrm{~kg} / \mathrm{m}^{2}([-0.80 ; 0.00])$ in TMGs+ patients (versus $0.06 \mathrm{~kg} / \mathrm{m}^{2}[-0.24$; $0.35]$ in CG patients), and $-0.53 \mathrm{~kg} / \mathrm{m}^{2}$ [-0.93; -0.14$]$ in TMGn+ patients, reflecting an anticipated respective difference of $-0.46 \mathrm{~kg} / \mathrm{m}^{2} \quad([-0.94 ; 0.02], \quad p=0.06)$ and $-0.57 \mathrm{~kg} / \mathrm{m}^{2} \quad([-1.05 ;-0.09], \quad p=0.02)$ relative to $\mathrm{CG}$ patients.

In patients with available waist circumference values, unadjusted $(n=206)$ and adjusted $(n=202)$ intergroup differences were $-1.67 \mathrm{~cm}$ $([-3.27 ;-0.06], \quad p=0.04)$ and $-1.29 \mathrm{~cm}$ ([-2.87;0.30], $p=0.11$ ), respectively, both in favour of the TMG. Intergroup differences were more pronounced in frequent users: $-2.00 \mathrm{~cm}$ $([-3.90 ;-0.10], p=0.04)$ between TMGs+ and CG patients and $-2.64 \mathrm{~cm}$ 


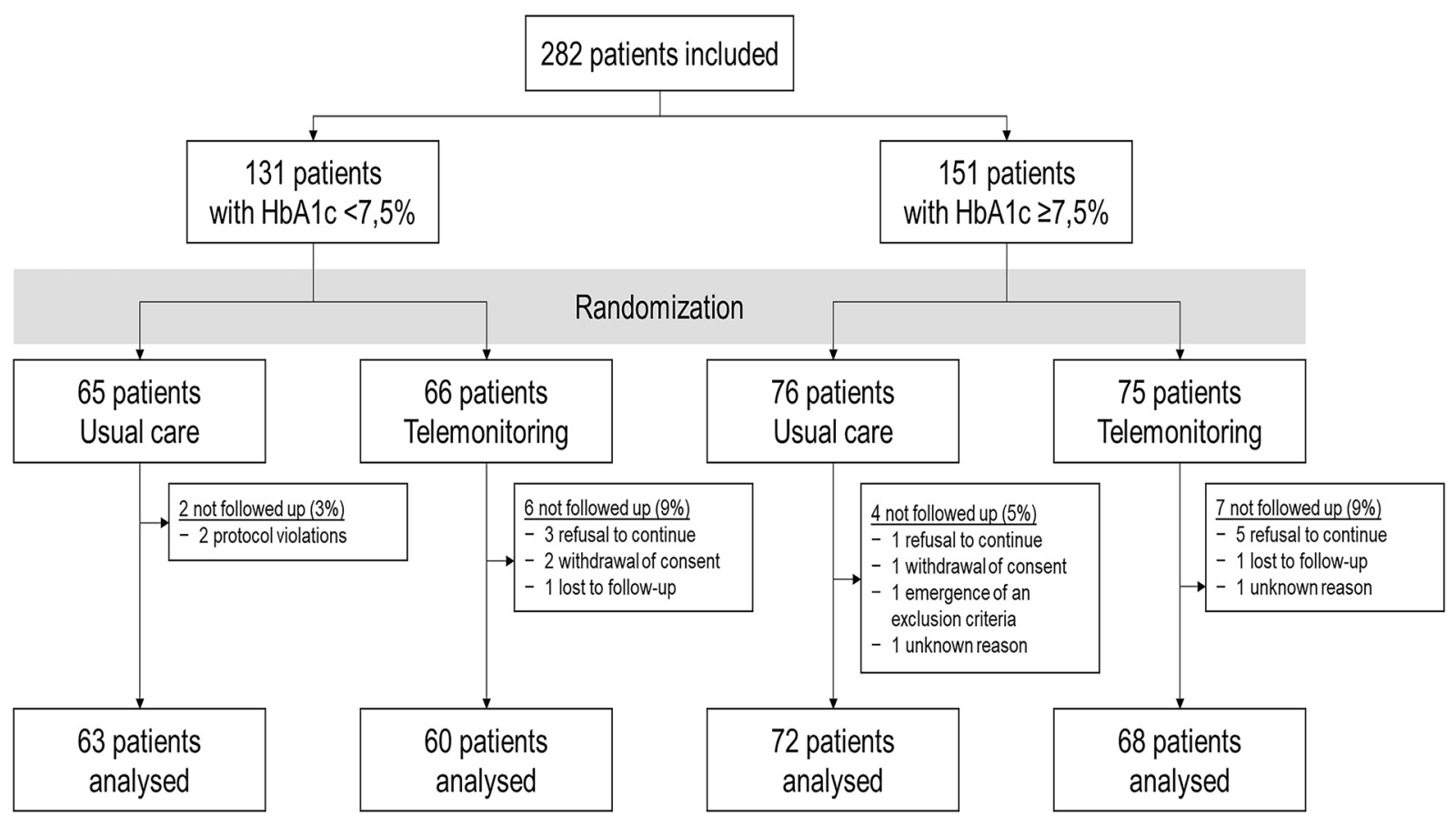

Fig. 1 Flow chart

([-4.60; -0.68$], \quad p=0.008) \quad$ between $\mathrm{TMGn}+$ and CG patients.

\section{Stratified Analyses According to Gender (Table 3)}

Predicted differences in HbA1c (primary endpoint $)$ were $-0.21 \%([-0.48 ; 0.05], p=0.12)$ in women and $-0.15 \% \quad([-0.35 ; 0.05]$, $p=0.15)$ in men, in favour of TMG patients.

Intergroup differences in BMI (TMG versus CG) were $-1.01 \mathrm{~kg} / \mathrm{m}^{2}([-1.79 ;-0.23], p=0.01)$ in women and $0.16 \mathrm{~kg} / \mathrm{m}^{2}([-0.27 ; 0.60], p=0.46)$ in men. According to the frequency of use, BMI differences of $-1.23 \mathrm{~kg} / \mathrm{m}^{2} \quad([-2.16 ;-0.31]$, $p=0.009)$ were documented in TMGs + women and $-1.40 \mathrm{~kg} / \mathrm{m}^{2}([-2.31 ;-0.48], p=0.003)$ in TMGn+ women. In terms of body weight, women in the TMG lost $2.7 \mathrm{~kg}([-4.79 ;-0.57], p=0.01)$ more than those in the CG group and up to $3.7 \mathrm{~kg}([-6.30 ;-1.11], p=0.005)$ in TMGs+ and $-3.78 \mathrm{~kg} \quad([-6.25 ;-1.31], p=0.003)$ in TMGn+ women. No significant changes in waist circumference were observed (data not shown).

\section{Correlation Between Device Use and Changes in HbA1c and BMI (Fig. 3)}

HbA1c significantly decreased over the 12-month intervention period as the number of connections to telemonitoring syntheses increased $(-0.16[-0.32 ;-0.003], p=0.04)$. No significant correlation was found between this frequency of use indicator and change in BMI $(p=0.28)$.

The number of connections to Nutri-Educ tended to be correlated with changes in HbA1c $(-0.15[-0.31 ; 0.02], p=0.08)$ and was significantly correlated with changes in BMI $(-0.30$ [-0.58; -0.02$], p=0.03)$.

\section{Device User Satisfaction (Fig. 4)}

At the end of the 12-month intervention period, $91.0 \%$ of telemonitored individuals completed the satisfaction questionnaire; $97.4 \%$ were completely satisfied or rather satisfied with device use and telemonitoring data synthesis. Very high levels of satisfaction (very satisfied or rather satisfied) were also recorded regarding the assistance provided by the device in terms of 
Table 1 Main baseline characteristics

\begin{tabular}{|c|c|c|c|c|c|c|}
\hline Gender & \multicolumn{2}{|c|}{ Total $(n=263)$} & \multicolumn{2}{|c|}{ CG $(n=135)$} & \multicolumn{2}{|c|}{ TMG $(n=128)$} \\
\hline Male & 166 & $63.1 \%$ & 84 & $62.2 \%$ & 82 & $64.1 \%$ \\
\hline Female & 97 & $36.9 \%$ & 51 & $37.8 \%$ & 46 & $35.9 \%$ \\
\hline \multicolumn{7}{|l|}{ Age (years) } \\
\hline Median (IIQ) & 60.6 & 13.0 & 60.3 & 14.0 & 61.3 & 12.4 \\
\hline Mean (SD) & 59.5 & 9.6 & 59.3 & 10.0 & 59.8 & 9.2 \\
\hline \multicolumn{7}{|l|}{ Randomisation stratum } \\
\hline Initial $\mathrm{HbA}_{\mathrm{lc}}<7.5 \%$ & 123 & $46.8 \%$ & 63 & $46.7 \%$ & 60 & $46.9 \%$ \\
\hline Initial $\mathrm{HbA}_{1 \mathrm{c}} \geq 7.5 \%$ & 140 & $53.2 \%$ & 72 & $53.3 \%$ & 68 & $53.1 \%$ \\
\hline \multicolumn{7}{|l|}{ Initial $\mathrm{HbA}_{1 \mathrm{c}}$} \\
\hline Median and IIQ & 7.6 & 1.1 & 7.7 & 1.1 & 7.6 & 1.2 \\
\hline Mean and standard deviation & 7.8 & 0.8 & 7.8 & 0.8 & 7.8 & 0.8 \\
\hline \multicolumn{7}{|l|}{ Body weight (kg) } \\
\hline Median and IIQ & 91.0 & 21.0 & 89.0 & 24.0 & 91.5 & 18.5 \\
\hline Mean and standard deviation & 91.7 & 16.7 & 90.0 & 16.0 & 93.5 & 17.3 \\
\hline \multicolumn{7}{|l|}{ BMI $\left(\mathrm{kg} / \mathrm{m}^{2}\right)$} \\
\hline Median and IIQ & 32.2 & 5.7 & 30.8 & 5.8 & 31.6 & 6.0 \\
\hline Mean and standard deviation & 31.8 & 5.3 & 31.2 & 5.2 & 32.5 & 5.4 \\
\hline \multicolumn{7}{|l|}{ Obesity (BMI $\geq 30 \mathrm{~kg} / \mathrm{m}^{2}$ ) } \\
\hline No & 99 & $37.8 \%$ & 58 & $43.6 \%$ & 41 & $32.0 \%$ \\
\hline Yes & 162 & $62.2 \%$ & 75 & $56.4 \%$ & 87 & $68.0 \%$ \\
\hline \multicolumn{7}{|l|}{ Waist circumference } \\
\hline Mean (SD) & 110.1 & 12.5 & 107.9 & 12.4 & 112.4 & 12.3 \\
\hline \multicolumn{7}{|l|}{ Duration of diabetes } \\
\hline Less than 5 years & 31 & $11.9 \%$ & 16 & $12.0 \%$ & 15 & $11.8 \%$ \\
\hline Between 5 and 15 years & 127 & $48.9 \%$ & 65 & $48.9 \%$ & 62 & $48.8 \%$ \\
\hline More than 15 years & 102 & $39.2 \%$ & 52 & $39.1 \%$ & 50 & $39.4 \%$ \\
\hline \multicolumn{7}{|l|}{ Diabetes complications } \\
\hline No & 121 & $46.5 \%$ & 60 & $45.1 \%$ & 61 & $48.0 \%$ \\
\hline Yes & 139 & $53.5 \%$ & 73 & $54.9 \%$ & 66 & $52.0 \%$ \\
\hline \multicolumn{7}{|l|}{ Details of complications } \\
\hline Retinopathy & 41 & $15.9 \%$ & 25 & $19.2 \%$ & 16 & $12.5 \%$ \\
\hline Nephropathy & 66 & $25.9 \%$ & 32 & $24.8 \%$ & 34 & $27.0 \%$ \\
\hline
\end{tabular}


Table 1 continued

\begin{tabular}{llllllr}
\hline & \multicolumn{2}{l}{ Total $(\boldsymbol{n}=\mathbf{2 6 3})$} & \multicolumn{2}{l}{ CG $(\boldsymbol{n}=\mathbf{1 3 5})$} & \multicolumn{2}{c}{ TMG $(\boldsymbol{n}=\mathbf{1 2 8})$} \\
\hline Peripheral neuropathy & 43 & $16.7 \%$ & 29 & $22.1 \%$ & 14 & $11.0 \%$ \\
Vegetative neuropathy & 10 & $4.0 \%$ & 6 & $4.7 \%$ & 4 & $3.2 \%$ \\
Coronary insufficiency & 47 & $18.4 \%$ & 19 & $14.7 \%$ & 28 & $22.2 \%$ \\
Cerebrovascular insufficiency & 12 & $4.7 \%$ & 8 & $6.1 \%$ & 4 & $3.2 \%$ \\
Lower limb artery disease & 25 & $9.8 \%$ & 9 & $6.9 \%$ & 16 & $12.9 \%$ \\
Chronic wound & 8 & $3.1 \%$ & 4 & 3.0 & 4 & $3.2 \%$ \\
Receiving insulin & & & & & & \\
No & 79 & $30.0 \%$ & 42 & $31.1 \%$ & 37 & $28.9 \%$ \\
Yes & 184 & $70.0 \%$ & 93 & $68.9 \%$ & 91 & $71.1 \%$ \\
\hline
\end{tabular}

Number and percentage, unless otherwise stated

improving their knowledge on diabetes (87.6\%) and nutrition (84.3\%). Device-generated assistance was deemed positive by $88.2 \%$ of subjects for the daily management of diabetes, by $81 \%$ for ensuring a balanced diet and by $85.5 \%$ for the practice of physical activity.

Fifty-five percentage of the investigators completed the satisfaction questionnaire; $85 \%$ of them reported having completely integrated the web application functions and over $80 \%$ found it easy to very easy to use, in terms of both patient records and telemonitoring synthesis reports. Finally, $82.3 \%$ were keen to continue using the device.

\section{DISCUSSION}

This randomised study shows that, compared to standard management including face-to-face outpatient visits, use of the EDUC@DOM telemonitoring and tele-education device for a period of 1 year did not significantly improve glycaemic control in people with type 2 diabetes (non-significant decrease in HbA1c levels, $p=0.06$ ). However, the metabolic benefit was logically correlated with the frequency of use of the device, which also resulted in weight loss and a reduction in waist circumference in women. It should be noted that individuals with T2D who participated in this study had all previously benefited from conventional education programmes and were all followed up by a diabetologist prior to inclusion, which could have minimised the overall impact of this intervention. The effectiveness of such connected programmes could thus certainly be increased in patients less experienced in the management of their disease.

The growing prevalence of $\mathrm{T} 2 \mathrm{D}$ along with the limited number of physicians in most countries makes it difficult to set up regular medical follow-up, sometimes requiring face-toface visits, especially when glycaemic control is insufficient. The recent development of telemonitoring devices and connected objects should change the way in which diabetes is managed and allow greater flexibility in terms of follow-up.

Nutritional intervention is effective in improving glycaemic control in T2D patients but it requires significant educational and therapeutic support from caregivers. In this specific field, advances in remote communication technologies and the development of 


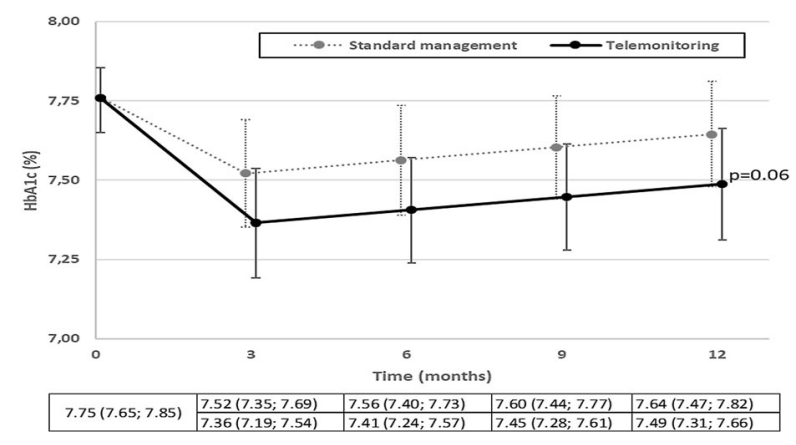

Fig. 2 Mean $\mathrm{HbA}_{1 \mathrm{c}}$ observed (at baseline) or predicted according to time of follow-up

applications for the automated assessment of nutritional intake have also opened up new avenues. However, the hypothesis that nutritional intervention based on telemedicine and artificial intelligence (AI) solutions could be as effective as or even more effective than traditional face-to-face consultations has yet to be demonstrated.

To date, studies evaluating telemonitoring devices dedicated to diabetes have generated mixed results in terms of both lifestyle and metabolic balance [18, 29-33]. By incorporating connected objects and tele-education tools including AI algorithms, the originality of our device is that it offers personalised support for lifestyle changes, particularly regarding nutrition.

Compliance with the use of the various connected objects and educational software is undoubtedly vital in ensuring the efficacy of this care method. Indeed, the benefit observed in terms of $\mathrm{HbA}_{1 \mathrm{c}}$ changes as well as changes in BMI in women depends on the frequency of connection to the educational software and patient access to telemonitoring data synthesis. In this context, it is important to emphasise the fact that, with a mean age of 60 years and rather persistent diabetes (of more than 5 years' duration in $88 \%$ of cases), our study participants were definitely not in the highly tech-savvy population.

In our study, we chose to include T2D patients regardless of their initial level of glycaemic control. Stratified analysis according to the baseline $\mathrm{HbA}_{1 \mathrm{c}}$ value did not highlight a significant difference between the two strata in terms of changes in $\mathrm{HbA}_{1 \mathrm{c}}$ over the 12-month follow-up period, although a greater drop could have been expected in less well-controlled individuals, as usually reported [34, 35]. However, from a public health perspective and to avoid long-term complications [36], we initially considered that, in addition to improving glycaemic control in individuals with high $\mathrm{HbA}_{1 \mathrm{c}}$ levels, maintaining subjects with slightly unbalanced diabetes at satisfactory $\mathrm{HbA}_{1 \mathrm{c}}$ levels is also valuable.

Weight and BMI monitoring also shows benefits directly related to the frequency of device use although this beneficial impact appears to be more significant in women than in men. A reduction in waist circumference is also correlated with device use but no gender effect is apparent. Such positive effects on body weight and fat distribution may have been associated with improved insulin sensitivity and a reduction in cardiovascular risk factors, but these parameters were not documented in our study [37].

In T2D, blood glucose monitoring must be linked to lifestyle interventions in order to improve metabolic control [32]. Therefore, in our telemonitoring and coaching approach, we included not only a glucose meter but also other connected objects and tele-education modules targeting diet and physical activity. It is important to note that the Nutri-Educ software included in our device has already demonstrated its effectiveness in improving dietary balance, weight and glycaemic control in individuals with diabetes or obesity [23, 24].

Long-term lifestyle changes remain a key challenge in the management of T2D to eradicate often deeply ingrained habits. To this end, telemonitoring and tele-education approaches offer innovative solutions through the provision of personalised and on-going advice to modify diet and physical activity practices while respecting patients' tastes and preferences. Such devices provide physicians and their patients with regular updates on assessments of the patients' lifestyle in relation to the jointly set targets. In addition, AI modules are now able to drive the solutions most suited to each patient's clinical profile. In addition to the data collected, the connected objects per se also play a 
Table $2 \mathrm{HbA}_{1 \mathrm{c}}$ changes according to randomisation group $(N=256)$ and frequency of device use

\begin{tabular}{|c|c|c|c|c|c|c|}
\hline & \multicolumn{3}{|l|}{ Models } & & \multicolumn{2}{|c|}{ Over 1 year } \\
\hline & $\bar{\beta}$ & 95\% CI & $p$ & & $\bar{\Delta}$ & 95\% CI \\
\hline \multicolumn{7}{|l|}{ Randomisation group } \\
\hline \multicolumn{7}{|l|}{ Control group (CG) } \\
\hline Telemonitoring group (TMG) & -0.16 & $-0.32 ; 0.01$ & 0.06 & * & -0.24 & $-0.42 ;-0.07$ \\
\hline \multicolumn{7}{|l|}{ Number of connections (synthesis) } \\
\hline Control group (CG) & ref & & & & -0.08 & $-0.26 ; 0.09$ \\
\hline TMGs - number $\leq$ median & -0.08 & $-0.28 ; 0.13$ & 0.45 & & -0.16 & $-0.38 ; 0.05$ \\
\hline TMGs+ number $>$ median & -0.23 & $-0.43 ;-0.03$ & 0.03 & $* *$ & -0.31 & $-0.53 ;-0.10$ \\
\hline \multicolumn{7}{|c|}{ Number of connections (Nutri-Educ) } \\
\hline Control group & ref & & & & -0.09 & $-0.26 ; 0.09$ \\
\hline TMGn - number $\leq$ median & -0.11 & $-0.31 ; 0.10$ & 0.30 & & -0.19 & $-0.40 ; 0.01$ \\
\hline TMGn + number $>$ median & -0.21 & $-0.41 ; 0.00$ & 0.05 & $* *$ & -0.29 & $-0.51 ;-0.07$ \\
\hline
\end{tabular}

Models adjusted for potential confounding factors defined a priori, taking into account time effect, centre effect and randomisation per stratum. HbAlc value at baseline was $7.75 \%(95 \% \mathrm{CI}[7.65 ; 7.85])$

With $\beta$ linear regression coefficients, 95\% CI 95\% confidence intervals, $p p$ value, $m$ average HbAlc observed at T0 (\%) and $\Delta$ predicted variation over 1 year

${ }^{* *}$ Significant difference between groups $(<0.05)$; ${ }^{*}$ difference at the limit of significance $(<0.10)$

pedagogical role by boosting motivation, but their impact is enhanced by the coaching effect in particular [31]. Finally, remote monitoring over several months prompts closer support and helps maintain the desired behavioural changes to control the disease. In line with this last point, most users were satisfied or very satisfied with the EDUC@DOM tool for assistance in diabetes management and especially in lifestyle changes, whether in terms of nutrition or physical activity.

Some study limitations must be acknowledged. First, blinding patients was not possible in this study, which might have resulted in an overestimation of the efficacy of the telemonitoring programme, as observed in other nonblind studies [38]. Moreover, the impact of being monitored according to the anticipated study design is an unavoidable part of the process and we cannot rule out the fact that patient behaviour may have been different in real-life settings. In addition, as in other randomised trials, the results might not be generalised to patients who would not have agreed to participate given their clinical or socio-economic profile (more serious medical history, poorer health, limited internet access, etc.). Finally, measuring satisfaction with a non-validated questionnaire might have led to less reliable measurements, making it more difficult to compare the results to other studies.

To date, the long-term impact of telemonitoring has not been assessed to any considerable extent in diabetes and, more generally, in chronic diseases. It would therefore be interesting to determine whether the positive trends reported in our 1-year study are maintained in the months following the cessation of telemonitoring. Further randomised studies should be carried out to establish optimal modalities for using telemonitoring devices in diabetes (focusing on subject profiles, ideal time for intervention, duration, combination or separation of telemonitoring and tele-education devices, involvement of specialist doctors and general practitioners, delegation of tasks to 
Table 3 Changes in body weight and BMI by group and device use, stratified on gender

\begin{tabular}{|c|c|c|c|c|c|c|c|c|}
\hline & \multicolumn{4}{|c|}{ Weight (in kg) } & \multicolumn{4}{|c|}{ BMI (in $\left.\mathrm{kg} / \mathrm{m}^{2}\right)$} \\
\hline & $\bar{\beta}$ & $95 \% \mathrm{CI}$ & $p$ & & $\bar{\beta}$ & 95\% CI & $p$ & \\
\hline \multicolumn{9}{|l|}{ In women $(N=86)$} \\
\hline \multicolumn{9}{|l|}{ Randomisation group } \\
\hline CG & ref & & & & ref & & & \\
\hline TMG & -2.68 & $-4.79 ;-0.57$ & 0.013 & ** & -1.01 & $-1.79 ;-0.23$ & 0.011 & ** \\
\hline \multicolumn{9}{|l|}{ Connection (synthesis) } \\
\hline CG & ref & & & & ref & & & \\
\hline TMGs- number $\leq$ median & -1.84 & $-4.46 ; 0.79$ & 0.170 & - & -0.75 & $-1.72 ; 0.22$ & 0.131 & - \\
\hline TMGs+ number $>$ median & -3.39 & $-5.87 ;-0.91$ & 0.007 & ${ }^{* *}$ & -1.23 & $-2.16 ;-0.31$ & 0.009 & ** \\
\hline \multicolumn{9}{|l|}{ Connections (Nutri-Educ) } \\
\hline CG & ref & & & & ref & & & \\
\hline TMGn - number $\leq$ median & -1.34 & $-3.98 ; 1.30$ & 0.319 & - & -0.54 & $-1.52 ; 0.44$ & 0.282 & - \\
\hline TMGn + number $>$ median & -3.78 & $-6.25 ;-1.31$ & 0.003 & ** & -1.40 & $-2.31 ;-0.48$ & 0.003 & ** \\
\hline \multicolumn{9}{|l|}{ In men $(N=150)$} \\
\hline \multicolumn{9}{|l|}{ Randomisation group } \\
\hline CG & ref & & & & ref & & & \\
\hline TMG & 0.46 & $-0.87 ; 1.80$ & 0.498 & - & 0.16 & $-0.27 ; 0.60$ & 0.463 & - \\
\hline \multicolumn{9}{|l|}{ Connection (synthesis) } \\
\hline CG & ref & & & & ref & & & \\
\hline TMGs- number $\leq$ median & 0.99 & $-0.64 ; 2.62$ & 0.233 & - & 0.34 & $-0.19 ; 0.88$ & 0.208 & - \\
\hline TMGs+ number $>$ median & -0.06 & $-1.68 ; 1.56$ & 0.945 & - & -0.01 & $-0.54 ; 0.52$ & 0.966 & - \\
\hline \multicolumn{9}{|l|}{ Connections (Nutri-Educ) } \\
\hline CG & ref & & & & ref & & & \\
\hline TMGn - number $\leq$ median & 0.91 & $-0.70 ; 2.52$ & 0.268 & - & 0.32 & $-0.21 ; 0.85$ & 0.234 & - \\
\hline TMGn + number $>$ median & -0.01 & $-1.65 ; 1.63$ & 0.987 & - & -0.001 & $-0.54 ; 0.54$ & 0.996 & - \\
\hline
\end{tabular}

Models adjusted for potential confounding factors defined a priori, taking into account time effect, centre effect and randomisation per stratum

With $\beta$ linear regression coefficients, $95 \%$ CI 95\% confidence intervals, $p p$ value

${ }^{*}$ Significant difference between groups $(<0.05),{ }^{*}$ difference at the limit of significance $(<0.10)$

other healthcare professionals, etc.). Further improvement of the user profile definition should facilitate the targeting of individuals best suited to this type of tool and should allow personalisation of the intervention modalities.

\section{CONCLUSION}

This study shows that at-home telemonitoring and tele-education did not significantly improve glycaemic control in T2D subjects 

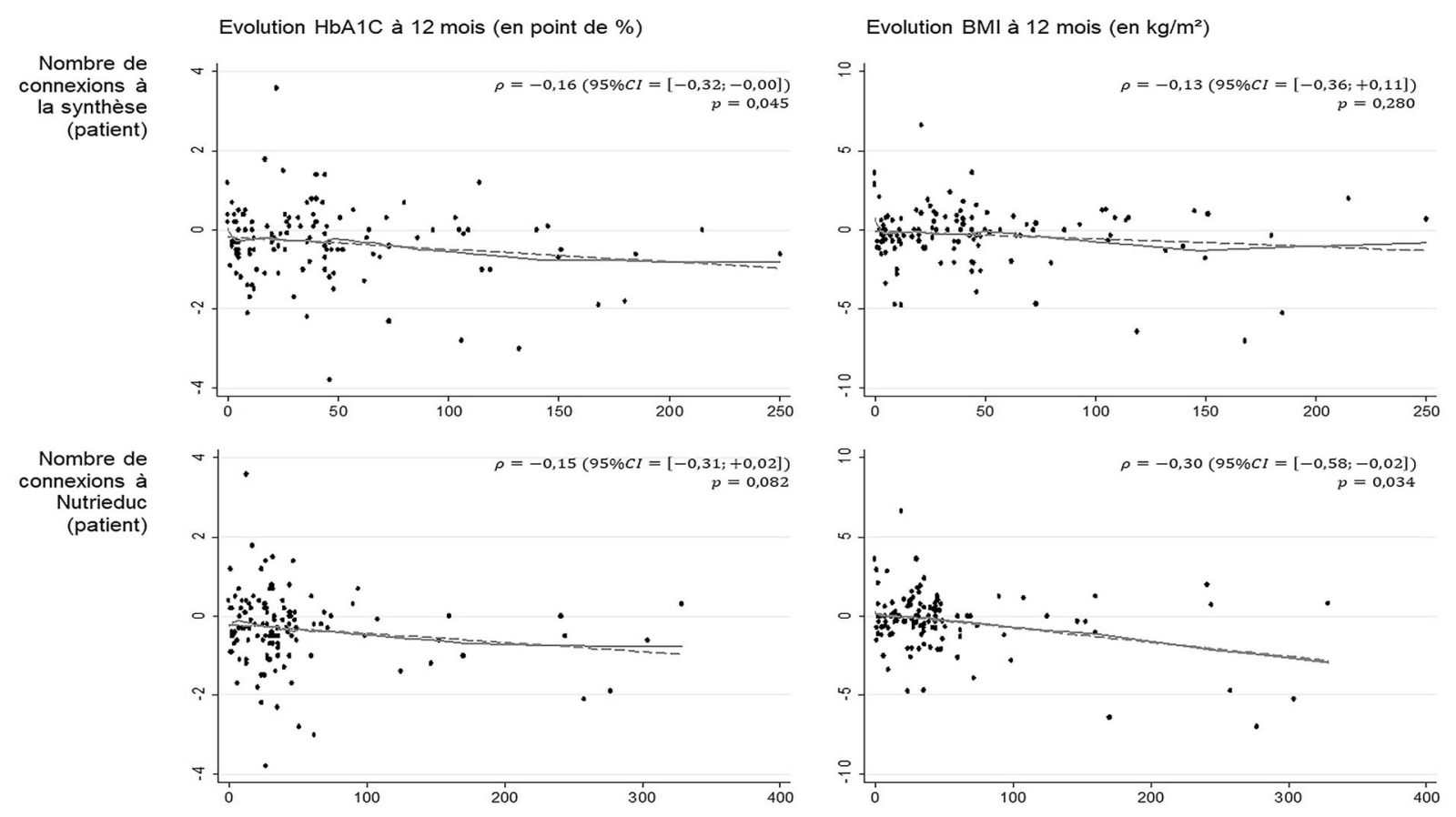

Fig. 3 Correlations between frequency of use and changes in $\mathrm{HbA}_{1 \mathrm{c}}$ and BMI. With: solid lines for non-parametric (Lowess) models and dashed lines for linear models. Since the correlations are graphically linear, the following are presented: $\rho$, Pearson correlation coefficient, with

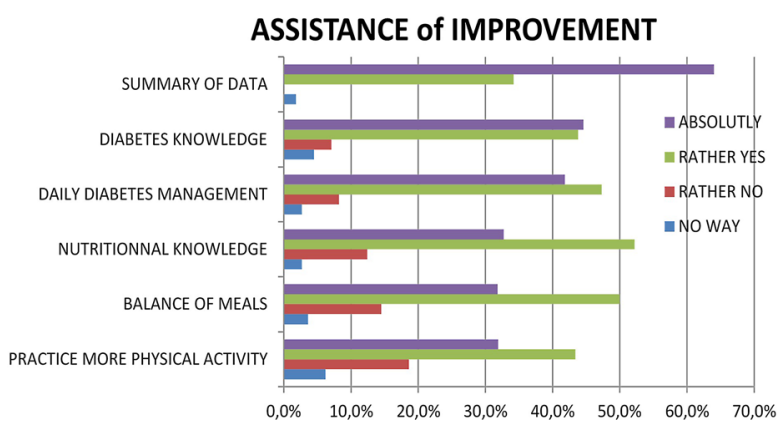

Fig. 4 Evaluation of device use by telemonitored patients

despite a slight, albeit significant decrease in HbA1c levels in frequent device users as well as significant weight loss in women only. This strategy could contribute to new organisational modalities for diabetes management, making it possible to respond to current public health issues while maintaining the relevance and quality of follow-up. By offering an alternative to the conventional healthcare system, confidence intervals estimated by bootstrapping (1000 replications) and $p$ value by the Pearson correlation coefficient test.

telemonitoring should allow the frequency of face-to-face medical consultations to be modulated according to individual requirements and availability, thus reducing healthcare costs. An economic analysis of the EDUC@DOM study after 2 years of follow-up will address the costsaving impact of this strategy.

\section{ACKNOWLEDGEMENTS}

We are very grateful to all patients who participated in this study. We also wish to extend our grateful thanks to the clinical teams at the University Hospital Centres of Grenoble, Lyon, Nantes and Nîmes, the Hospital Centres of Cahors, Corbeil-Essonne, Lannemezan and Tarbes, the Ambroise-Paré (Toulouse), Claude Bernard (Albi) and Pasteur (Toulouse) clinics and our two colleagues working in private practices. 
Funding. This study and the journal's Rapid Service Fee has been funded by the French Ministry of Health [call for projects for research programmes on the performance of the healthcare system (PREPS 2012 no. 12-0180078)] and the Midi-Pyrénées Regional Council [Call for Projects for Research on Clinical Transfer and Standardization in Bio-health 2012, year 1 (no. 11052617) and year 2 (no. 13050630)] for the development of the study methodology, data collection, data analysis and interpretation and the publication of the results. We are grateful to the SANOFI Laboratory for its participation and provision of blood glucose monitors.

Authorship. All of the named authors meet the International Committee of Medical Journal Editors' (ICMJE) criteria for authorship for this article, take responsibility for the integrity of the work as a whole, and have given their approval for this version to be published.

Authors' Contributions. M.C. Turnin, S. Schirr-Bonnans and JC Buisson contributed to the creation and design of the study. M.C. Turnin, P. Gourdy, J. Martini, M.C. Chauchard, M.F. Poncet, V. Cosma, S. Lablanche, M. Coustols-Valat, L. Chaillous, C. Thivolet, C. Sanz, A. Penfornis, J. Delaunay, S. Schirr-Bonnans and S. Taoui participated in the recruitment and follow-up of patients enrolled in the study. B. Lepage and H. Colineaux developed the overall study methodology and statistical methods. L. Molinier, M. Mounié and N. Costa developed the methodology for the healtheconomic assessment. M.C. Turnin, P. Gourdy, B. Lepage, H. Colineaux and H. Hanaire contributed to the analysis of the results and wrote the paper. All authors read and approved the final manuscript.

List of Investigators. EDUC@DOM study group includes all investigators involved in the study: Prof. P.Y. Benhamou and Dr S. Lablanche (CHU de Grenoble - University Hospital Centre), Prof. C. Thivolet (Hospices Civils de Lyon Lyon Civil Hospices), Dr L. Chaillous (CHU de Nantes), Dr M. Rodier and V. Cosma (CHU de Nîmes), Prof. A. Penfornis (Sud Francilien
Hospital Centre), Dr F. Ayon and F. Puel-Olivier (Cahors Hospital Centre), Dr S. Fontaine (Hôpital Ducuing-Toulouse), Doctor M. Perron (Lannemezan Hospital Centre), Dr J. Arrivié (Tarbes Hospital Centre), Dr M. Coustols-Valat and F. Cousty-Pech (Clinique Ambroise-Paré-Toulouse), Dr M.F. Poncet (Clinique Claude Bernard- Albi), Dr C. Sanz and C. Rouby (Clinique Pasteur-Toulouse), Dr F. Lafon (General practitioner, Toulouse), Dr I. Moura (Diabetologist, Gaillac).

Prior Presentation. Assessment of the effectiveness of a home telemonitoring programme and its cost in the care of type 2 diabetic patients recruited from a healthcare network. SFD Telemedicine and Innovative Technologies, SFD Congress, France, Lyon, March 22 to 25, 2016. First results of the EDUC@DOM multicentre protocol for telemonitoring Type 2 Diabetes. SFD Telemedicine and Innovative Technologies, SFD Congress, France, Nantes, March 20 to 23, 2018. First results of the EDUC@DOM multicentre protocol for telemonitoring Type 2 Diabetes. SFE Congress, France, Nancy, September 12 to 14, 2018.

Disclosures. Marie-Christine Turnin has received congress invitations and consultancy fees from VitalAire/Air Liquide Health, Eli Lilly, and Sanofi. Pierre Gourdy has received advisory board and speaker honoraria from Abbott, Amgen, AstraZeneca, Novo Nordisk, Boehringer Ingelheim, Eli Lilly, Merck Sharp \& Dohme, Mundipharma, Sanofi, and Servier. Jacques Martini has nothing to disclose. Jean-Christophe Buisson has nothing to disclose. MarieChristine Chauchard has nothing to disclose. Jacqueline Delaunay has received congress invitations and consultancy fees from Sanofi. Solène Schirr-Bonnans has nothing to disclose. Soumia Taoui has nothing to disclose. MarieFrance Poncet has nothing to disclose. Valeria Cosma has nothing to disclose. Sandrine Lablanche has nothing to disclose. Magali Coustols-Valat has nothing to disclose. C Thivolet has received travel support for meetings, speaker fees and honoraria from Abbott Diabetes Care, Janssen, Lilly, Novo-Nordisk, Medtronic, Roche Diabetes Care, and Sanofi and is 
an advisory board member for Insulet and Medtronic. Caroline Sanz has nothing to disclose. Alfred Penfornis has nothing to disclose. Benoît Lepage has nothing to disclose. Hélène Colineaux has nothing to disclose. Michaël Mounié has nothing to disclose. Nadège Costa has nothing to disclose. Laurent Molinier has nothing to disclose. Hélène Hanaire has received congress invitations, honoraria and consultancy fees from Abbott, Animas/Johnson \& Johnson, Medtronic, Roche, Eli Lilly, Novo Nordisk and MSD, and served on advisory board panels for Diabeloop and Insulet.

Compliance with Ethics Guidelines. All type 2 diabetes patients participating in this research received verbal and written information and were given the opportunity to ask any questions to help them understand the study. They signed a voluntary informed consent form before the research began. This study was submitted to the South-West and French Overseas Territories' Ethics Committee and received a favourable opinion from the EC on 27/05/2013. This study was performed in accordance with the Helsinki Declaration of 1964 and its later amendments.

Data Availability. The datasets generated and/or analysed during the current study are available from the corresponding author on reasonable request.

Open Access. This article is licensed under a Creative Commons Attribution-NonCommercial 4.0 International License, which permits any non-commercial use, sharing, adaptation, distribution and reproduction in any medium or format, as long as you give appropriate credit to the original author(s) and the source, provide a link to the Creative Commons licence, and indicate if changes were made. The images or other third party material in this article are included in the article's Creative Commons licence, unless indicated otherwise in a credit line to the material. If material is not included in the article's Creative Commons licence and your intended use is not permitted by statutory regulation or exceeds the permitted use, you will need to obtain permission directly from the copyright holder. To view a copy of this licence, visit http://creativecommons.org/licenses/by$\mathrm{nc} / 4.0 /$.

\section{REFERENCES}

1. Davies MJ, D'Alessio DA, Fradkin J, et al. Management of hyperglycemia in type 2 diabetes, 2018. A consensus report by the American Diabetes Association (ADA) and the European Association for the Study of Diabetes (EASD). Diabetes Care. 2018;2018(41):2669-701.

2. Diabetes Control and Complications Trial Research Group. The effect of intensive treatment of diabetes on the development and progression of long-term complications in insulin-dependent diabetes mellitus. N Engl J Med. 1993;329:997-1086.

3. UK Prospective Diabetes Study (UKPDS) Group. Intensive blood glucose control with sulphonylureas or insulin compared with conventional treatment and risk of complications in personnes with type 2 diabetes (UKPDS 33). Lancet. 1998;352: 837-53.

4. Trento M, Gamba S, Gentile L, et al. Rethink Organization to iMprove Education and Outcomes (ROMEO). A multicenter randomized trial of lifestyle intervention by group care to manage type 2 diabetes. Diabetes Care. 2010;33:745-7.

5. Izquierdo R, Lagua CT, Meyer S, et al. Telemedicine intervention effects on waist circumference and body mass index in the IDEATel project. Diabetes Technol Ther. 2010;12:213-20.

6. Wootton R. Twenty years of telemedicine in chronic disease management - an evidence synthesis. J Telemed Telecare. 2012;18:211-20.

7. Siriwardena LS, Wickramasinghe WA, Perera KL, et al. A review of telemedicine interventions in diabetes care. J Telemed Telecare. 2012;18:164-8.

8. Holtz B, Lauckner C. Diabetes management via mobile phones: a systematic review. Telemed eHealth. 2012;18:175-84.

9. Marcolino MS, Maia JX, Alkmim MB, et al. Telemedicine application in the care of diabetes patients: Systematic review and meta-analysis. PLoS ONE. 2013;8:e79246.

10. Holmen $\mathrm{H}$, Torbjornsen $\mathrm{A}$, Wahl AK, et al. A mobile health intervention for self-management and lifestyle change for persons with type 2 diabetes, part 2: one-year results from the norvegian randomized 
controlled trial RENEWING HEALTH. JMIR MHealth Uhealth. 2014;2:e57.

11. Rasmussen OW, Lauszus FF, Loekke M. Telemedicine compared with standard care in type 2 diabetes mellitus: a randomized trial in an outpatient clinic. J Telemed Telecare. 2016;22(6):363-8. https://doi.org/10.1177/1357633X15608984 (Epub 2015 Oct 14. PMID: 26468213).

12. Wang G, Zhang Z, Feng Y, Sun L, Xiao X, Wang G, Gao Y, Wang H, Zhang H, Deng Y, Sun C. Telemedicine in the management of type 2 diabetes mellitus. Am J Med Sci. 2017;353(1):1-5. https:// doi.org/10.1016/j.amjms.2016.10.008 (Epub 2016 Oct 27. PMID: 28104096).

13. Fu H, McMahon SK, Gross CR, Adam TJ, Wyman JF. Usability and clinical efficacy of diabetes mobile applications for adults with type 2 diabetes: a systematic review. Diabetes Res Clin Pract. 2017;131: 70-81. https://doi.org/10.1016/j.diabres.2017.06. 016 (Epub 2017 Jun 21. PMID: 28692830).

14. Lee SWH, Chan CKY, Chua SS, Chaiyakunapruk N. Comparative effectiveness of telemedicine strategies on type 2 diabetes management: a systematic review and network meta-analysis. Sci Rep. 2017;7(1):12680. https://doi.org/10.1038/s41598017-12987-z (PMID: 28978949; PMCID: PMC5627243).

15. Bollyky JB, Bravata D, Yang J, Williamson M, Schneider J. Remote lifestyle coaching plus a connected glucose meter with certified diabetes educator support improves glucose and weight loss for people with type 2 diabetes. J Diabetes Res. 2018;16(2018): 3961730. https://doi.org/10.1155/2018/3961730 (PMID: 29888288; PMCID: PMC5977036).

16. Lee PA, Greenfield G, Pappas Y. The impact of telehealth remote patient monitoring on glycemic control in type 2 diabetes: a systematic review and meta-analysis of systematic reviews of randomised controlled trials. BMC Health Serv Res. 2018;18(1): 495. https://doi.org/10.1186/s12913-018-3274-8 (PMID: 29940936; PMCID: PMC6019730).

17. Jeong JY, Jeon JH, Bae KH, Choi YK, Park KG, Kim JG, Won KC, Cha BS, Ahn CW, Kim DW, Lee CH, Lee IK. Smart care based on telemonitoring and telemedicine for type 2 diabetes care: multi-center randomized controlled trial. Telemed J EHealth. 2018;24(8):604-13. https://doi.org/10.1089/tmj. 2017.0203 (Epub 2018 Jan 17. PMID: 29341843).

18. De Vasconcelos HCA, Lira Neto JCG, de Araújo MFM, Carvalho GCN, de Souza Teixeira CR, de Freitas RWJF, Damasceno MMC. Telecoaching programme for type 2 diabetes control: a randomised clinical trial. Br J Nurs. 2018;27(19):1115-20. https://doi.org/10.12968/bjon.2018.27.19.1115

(PMID: 30346819).

19. Von Storch K, Graaf E, Wunderlich M, Rietz C, Polidori MC, Woopen C. Telemedicine-assisted selfmanagement program for type 2 diabetes patients. Diabetes Technol Ther. 2019;21(9):514-21. https:// doi.org/10.1089/dia.2019.0056 (Epub 2019 Jul 9. PMID: 31287736).

20. Ruiz de Adana MS, Alhambra-Expósito MR, MuñozGarach A, et al. Randomized study to evaluate the impact of telemedicine care in patients with type 1 diabetes with multiple doses of insulin and suboptimal HbA1c in Andalusia (Spain): PLATEDIAN study. Diabetes Care. 2020;43(2):337-42.

21. Turnin MC, Schirr-Bonnans S, Martini J, et al. Educ@dom: comparative study of the telemonitoring of patients with type 2 diabetes versus standard monitoring-study protocol for a randomized controlled study. Diabetol Metab Syndr. 2017;9:52.

22. Buisson JC. Nutri-Educ, a nutrition software application for balancing meals, using fuzzy arithmetic and heuristic search algorithms. Artif Intell Med. 2008;42:213-27.

23. Buisson JC, Garel A. Balancing meals using fuzzy arithmetics and heuristic search algorithms. IEEE Trans Fuzzy Syst. 2003;11:68-78.

24. American Diabetes Association. Clinical practices recommendations 2010. Standards of medical care in diabetes-2012. Diabetes Care. 2012;35(Supplement 1):S11-63.

25. Turnin MC, Beddok R, Clottes J, et al. Telematic expert system DIABETO: new tool for diet selfmonitoring for diabetic patients. Diabetes Care. 1992;15:204-12.

26. Turnin MC, Bolzonella Pene C, Dumoulin S, et al. Multicenter evaluation of the Nutri-Expert Telematic System in diabetic patients. Diabete Metab. 1995;21:26-33.

27. Turnin MC, Bourgeois O, Cathelineau G, et al. Multicenter randomized evaluation of a nutritional education software in obese patients. Diabetes Metab. 2001;27:139-47.

28. Frison L, Pocock SJ. Repeated measures in clinical trials: analysis using mean summary statistics and its implications for design. Stat Med. 1992;11: $1685-704$.

29. Schoeppe S, Alley S, Van Lippevelde W, et al. Efficacy of interventions that use apps to improve diet, physical activity and sedentary behaviour: a systematic review. Int J Behav Nutr Phys Act. 2016;13: 127. 
30. Casimatis M, Kavanagh D. Effects of type 2 diabetes behavioural telehealth interventions on glycaemic control and adherence: a systematic review. J Telemed Telecare. 2012;0:1-8.

31. Cho JH, Chang SA, Kwon HS, et al. Long-term effect of the Internet based glucose monitoring system on HbA1c reduction and glucose stability: a 30-month follow-up study for diabetes management with a ubiquitous medical care system. Diabetes Care. 2006;29:2625-31.

32. Montori VM, et al. The impact of planned care and a diabetes electronic management system on community-based diabetes care: the Mayo Health System Diabetes Translation Project. Diabetes Care. 2002;25:1952-7.

33. Block G, Azar K, Romanelli R, et al. Diabetes prevention and weight loss with a fully automated behavioral intervention by email, web and mobile phone: a randomized controlled trial among persons with prediabetes. J Med Internet Res. 2015;17: e240.

34. Charpentier G, Benhamou PY, Dardari D, et al. The diabeo software enabling individualized insulin dose adjustments combined with telemedicine support improves $\mathrm{HbA}_{1 \mathrm{c}}$ in poorly controlled type 1 diabetic patients. Diabetes Care. 2011;34:533-9.

35. Quinn CC, Shardell MC, Terrin ML, et al. Clusterrandomized trial of mobile phone personalized behavioral intervention for blood glucose control. Diabetes Care. 2011;34:1934-42 (erratum in: Diabetes care 2013; 36 (11): 3850).

36. Farmer AJ, Prevost AT, Hardeman W, et al. Protocol for SAMS (support and advice for medication study: a randomized controlled trial of an intervention to support patients with type diabetes with adherence to medication). BMC Fam Pract. 2008;9:20.

37. Seo D, Niu J. Evaluation of internet-based interventions on waist circumference reduction: A metaanalysis. J Med. Internet. Res. 2015;17(7):e181. https://doi.org10.2196/jmir.3921.

38. Hróbjartsson A, Emanuelsson F, Skou Thomsen AS, Hilden J, Brorson S. Bias due to lack of patient blinding in clinical trials. A systematic review of trials randomizing patients to blind and nonblind sub-studies. Int J Epidemiol. 2014;43(4):1272-83. https://doi.org/10.1093/ije/dyu115

24881045. PMCID: PMC4258786).
(PMID: 